\title{
Self-compassion and the Mitigation of Negative Affect in the Era of Social Distancing
}

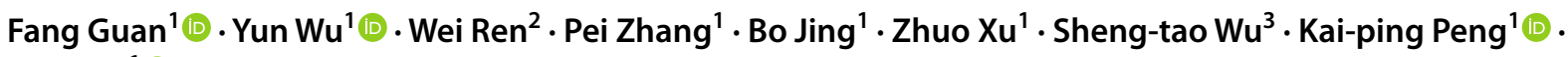 \\ Ji-bo $\mathrm{He}^{1}$ (1)
}

Accepted: 15 June 2021 / Published online: 28 June 2021

(c) The Author(s), under exclusive licence to Springer Science+Business Media, LLC, part of Springer Nature 2021

\begin{abstract}
Objectives The outbreak of contagious diseases and their associated non-pharmaceutical interventions can lead to negative mental health consequences. This study aimed to investigate online self-compassion exercises' effectiveness in alleviating people's negative affect (anxiety and negative emotions) during the COVID-19 pandemic lockdown.

Methods Study 1 examined the associations between self-compassion and negative affect using an anonymous online survey. In study 2 , two pilot experiments were conducted to examine online self-compassion exercises' (i.e., instructor-guided meditation and self-guided writing) effectiveness to mitigate individuals' negative affect. In study 3 , a randomized controlled study was conducted to further examine online self-compassion writing's effectiveness in reducing people's negative affect. Results The results of study 1 indicated a significant negative association between self-compassion and participants' negative affect. Participants in study 2a reported significant decreases in negative affect after completing the self-compassion meditation. Study $2 \mathrm{~b}$ showed that participants who completed the self-compassion writing reported significantly more selfcompassion and less anxiety when compared to participants who did not. Study 3 showed substantial pre/post-test changes in participants who completed self-compassion writing. Importantly, there were significant increases in participants' selfcompassion and decreases in negative affect when compared to participants in the control condition.

Conclusions Self-compassion exercises were effective to alleviate individuals' anxiety and reduce negative emotions (even within a brief session) during the pandemic lockdown.
\end{abstract}

Keywords Self-compassion $\cdot$ Anxiety $\cdot$ Negative emotions $\cdot$ Telehealth $\cdot$ COVID-19

Since the beginning of 2020, the outbreak of coronavirus disease 2019 (COVID-19) has significantly influenced the health and lives of people globally from many perspectives. Not only the pandemic itself (as a highly contagious disease) is threatening, but also the severe measures taken to contain the virus are also frustrating and handicapping to most of the people involved. Lessons from prior experiences on similar crises (e.g., H1N1 influenza, severe acute respiratory syndrome) have shown that such public health

Kai-ping Peng

pengkp@mail.tsinghua.edu.cn

1 Department of Psychology, Tsinghua University, Beijing, China

2 The Department of Youth and Children Work, China Youth University of Political Studies, Beijing, China

3 School of Sociology and Anthropology, Xiamen University, Xiamen, China events can trigger substantial emotional distress in individuals (Mak et al., 2009; Ofri, 2009). The feeling of emotional distress (i.e., negative affect including anxiety, fear, sadness, and other unpleasant emotions) has been found to be one of the primary causes of individuals' substandard level of psychological and physical well-being (Dua, 1993; Grinde, 2015). There is now increasing evidence indicating that negative emotional experiences, especially anxiety, are highly prevalent among the general public in the current pandemic (Huang \& Zhao, 2020; Mann et al., 2020; Petzold et al., 2020; Rajkumar, 2020; Wang et al., 2020). Take the United States (U.S.) as an example; big data showed that the general public's emotion on Twitter maintained a stable fluctuation within positive ranges before the pandemic outbreak. However, there was a drastic fluctuation in the general public's emotion index 1 month after the pandemic outbreak, suggesting the public's reaction toward the pandemic becoming 
more emotionally negative (see Appendix A in the supplementary materials).

It is pressing and vital to identify the psychological factors that can mitigate the negative affect of the general public and deliver effective intervention methods that equip people with skills to cope with the pandemic's negative emotional experiences. Many researchers have suggested that intrapersonal resources, such as self-compassion, may be a protective factor for the general public to cope with difficult emotional experiences during the current pandemic (Coyne et al., 2020; Gupta et al., 2020; Jiménez et al., 2020; Kavakli, 2020; Lau et al., 2020). Drawing from Eastern philosophy that originated from Buddhism, Neff (2003b) introduced self-compassion as a healthy self-attitude to Western psychology. Neff conceptualized self-compassion as a coping and emotional regulation strategy of individuals in times and situations of suffering, failures, perceived inadequacy, or general life difficulties (Neff et al., 2020). Neff suggested that compassionate self-responding to personal struggles involves three main components: self-kindness (i.e., extending kindness and understanding to oneself), common humanity (i.e., seeing one's experience as part of a larger human experience), and mindfulness (i.e., holding one's painful thoughts and feelings in balanced awareness). People who hold high self-compassion are often characterized as being caring and compassionate towards themselves in the face of hardship, suffering, or perceived inadequacy. Since then, there has been a substantial increase in research studies investigating the relations between self-compassion and individuals' psychological well-being (Neff \& Dahm, 2017; Nelson et al., 2016; Raes, 2011).

A meta-analysis conducted by Zessin et al. (2015) reviewed 79 studies that were published up to 2015 and provided empirical support of moderate-to-strong associations between self-compassion and various forms of wellbeing (e.g., psychological, cognitive, affective). With consistent relations between self-compassion and individuals' well-being being established in the literature, researchers have been turning their attention to explore whether selfcompassion skills can be taught (Barnard \& Curry, 2011; Neff \& Dahm, 2017). In particular, past studies have shown that self-compassion-based interventions can successfully cultivate peoples' self-compassion level and promote individuals' emotional well-being by reducing negative emotions, anxiety, and depression (Bluth \& Eisenlohr-Moul, 2017; Dundas et al., 2017; Smeets et al., 2014; Wilson et al., 2019). Despite the effectiveness of these intervention programs, in practice, only a small proportion of individuals with poor emotional well-being can and do receive proper care (Andrade et al., 2014; Mojtabai et al., 2011; Wang et al., 2007). Time constraints, financial difficulties, lack of mental health resources, desires to handle the issues by oneself, concerns about stigma, and privacy are common barriers that discourage people from seeking mental health care (Andrade et al., 2014; Beshai et al., 2020). These common barriers are enough of a challenge before the pandemic outbreak. With the scale of the pandemic continually expanding and the implementation of social distancing measures leading to substantial impacts on individuals' lives (e.g., mobility restriction, economic burdens), the need for improved access to psychological programs became even more pressing.

To address the accessibility issues and reduce the risk of individuals being infected while providing/receiving care during the pandemic, researchers and healthcare providers proposed that telehealth delivery of the treatment programs may be an ideal solution to maximize public health outcomes (Racine et al., 2020; Whaibeh et al., 2020). Telehealth delivery of mental health programs often involves the use of information and communication technologies (e.g., videoconferencing, phone) to evaluate, manage, and treat people's psychological symptoms (Langarizadeh et al., 2017). A few pilot studies have examined the feasibility and effectiveness of online forms of self-compassion-based interventions and provided support to the benefit of such programs on reducing people's distress and promoting better well-being (Finlay-Jones et al., 2017; Halamová et al., 2018; Krieger et al., 2019; Rao \& Kemper, 2016).

However, it is worth noting that the research studies that investigated self-compassion and individuals' emotional well-being have been mainly conducted in Western industrialized societies (Wilson et al., 2019; Zessin et al., 2015). Though the concept of self-compassion was drawn from Buddhism philosophy, relatively few research studies have been carried out in East Asian countries (where Buddhism is the most prevalent religious affiliation). There is a lack of empirical evidence on self-compassion's associations with and the effectiveness of its related interventions on emotional well-being in Eastern Asian countries like China. Given the cultural differences among countries and for meeting the general public's emotional and psychological needs in the current pandemic, it is vital to explore evidencebased psychological treatments that are scalable and easy to administer under the condition of social distancing implementation (Beshai et al., 2020).

Therefore, the main aims of this paper were (1) to establish a relation between self-compassion and negative affect (anxiety and negative emotions) among Chinese citizens, and (2) to investigate the potential utility of online self-compassion exercises on reducing the general public's negative affect in the context of the pandemic lockdown. Specifically, we examined the associations between individuals' selfcompassion and their negative emotions and anxiety (study 1). Then, we assessed the effectiveness of two pilot online self-compassion exercises-the instructor-guided meditation (study 2a) and the self-guided writing (study $2 \mathrm{~b}$ ) -in alleviating and reducing individuals' anxiety and negative 
emotions. Last, based on the results of study $2 \mathrm{~b}$, we conducted a randomized controlled study to improve further its experimental method for a more accurate examination of the effectiveness of the self-compassion writing exercise (study 3 ).

\section{Study 1}

Prior studies have demonstrated that self-compassionate individuals tend to have better psychological health and lower levels of anxiety and depression (Zessin et al., 2015). However, there is a lack of empirical evidence on the relations between self-compassion and individuals' emotional well-being among the general public in China. Thus, before examining the effectiveness of self-compassion-based exercises on reducing the negative affect in Chinese citizens, we first examined the associations between self-compassion and negative affect in the pandemic context. We hypothesized that participants who scored high on self-compassion would report significantly lower anxiety levels and fewer feelings of negative emotions.

\section{Method}

\section{Participants}

There were 314 participants from the general population who responded to the survey. After excluding participants who were under 18 years of age $(n=26)$ and who were resided overseas at the time of data collection $(n=4)$, the sample comprised 288 Chinese participants (105 males, 183 females). Participants ranged between 18 and 58 years old $\left(M_{\text {age }}=33.74, S D=10.59\right)$. The majority of the participants were well-educated, with $86.6 \%$ reported holding at least an undergraduate degree. Of the participants included, $73.6 \%$ of participants reported being employed/self-employed, and the rest were full-time students. All participants reported following the stay-at-home instruction at the time of data collection, with $88.4 \%$ reported staying with their families and $11.6 \%$ were living alone. In addition, all participants except 16 reported that the pandemic has led to varying degrees of impact on their lives. Specifically, a large proportion of participants indicated that the pandemic had impacted their daily routines $(72.2 \%)$ and work $(64.8 \%)$, followed by study (40.5\%), emotional well-being (35.9\%), relationships (18.7\%), and families (10.2\%).

\section{Procedure}

The data were collected at the end of March 2020. Following ethical approval, the study was advertised on China's two largest social media platforms (i.e., WeChat and QQ).
Participants were informed that they would need to complete a multi-section questionnaire and that doing so would take approximately 10-15 min. After providing informed consent, the participants completed the SCS, PANAS, and SAS. Participants who completed the survey were thanked and received monetary compensation (¥10) for their participation. Details for relevant counseling services were also provided at the beginning and the end of the survey.

\section{Measures}

Self-compassion The 26-item self-compassion scale (SCS; Neff, 2003a, 2016) was used to assess the participants' thoughts, emotions, and behaviors associated with selfcompassion. The SCS examines the three core components of self-compassion (i.e., self-kindness, common humanity, mindfulness) and their negative counterparts in daily life (i.e., self-judgment, isolation, and over-identification). Participants rated each item on a 5-point Likert scale, ranging from $1=$ "almost never" to $5=$ "almost always." An example item includes "I' $m$ tolerant of my own flaws and inadequacies." To calculate an overall compassion score, items representing the uncompassionate responses were first reversecoded. Then, the mean score of each subscale was used to compute a total mean. The Chinese version of the SCS has been demonstrated to be a reliable and valid measure in the Chinese populations (Cronbach's $\alpha=0.84$ ) (Yang et al., 2016). The internal consistency was also excellent in the current study (Cronbach's $\alpha=0.90$ ).

General Anxiety The 20-item self-rating anxiety scale (SAS; Zung, 1971) was used to examine participants' general anxiety level. The SAS assesses a variety of anxiety symptoms, including both psychological (e.g., "I feel afraid for no reason at all") and somatic symptoms (e.g., "I feel my heart beating fast"). Participants were asked to describe how they have been feeling during the past month. Each item was rated on a 4-point scale, which $1=$ "none or a little of the time" and $4=$ "most or all of the time." To generate an overall anxiety score, items representing positive experiences (e.g., I fall asleep easily and get a good night's sleep") were reverse-coded. Then, all items were summed to compute a total score, with a higher score indicating participants' higher level of anxiety. Cronbach's $\alpha$ was 0.84 for the SAS in the current study. Raw scale scores for the SAS range from 20 to 80 , with a recommended raw score of 36 as a cut-off point to indicate whether a participants' anxiety level reached clinical significance (Dunstan \& Scott, 2020). The Chinese version of SAS used in the current study was translated by $\mathrm{Wu}(1990)$, and the internal consistency was good (Cronbach's $\alpha=0.84$ ). 
Negative Emotions The positive and negative affect schedule (PANAS; Watson et al., 1988) was used to examine participants' negative moods or affective states. The PANAS contains two 10-item subscales, each assessing individuals' positive states and negative states (e.g., distressed, irritable). Given the purpose of this study, only the negative emotion subscale (PANAS-NA) was used. Participants were asked to rate the extent to which they "generally" feel each emotion during the last month. Each item was rated on a 5-point Likert scale, ranging from $1=$ "very slightly or not at all" to $5=$ "extremely." The total score for both subscales can range from 10 to 50 , with higher scores indicating a higher experience of negative emotions. The internal consistency for negative emotions was excellent in the current sample $(\alpha=0.90)$. The emotion words in PANAS-NA were translated and back-translated by two bilingual research assistants to suit the Chinese-speaking participants' needs.

\section{Data Analyses}

Data analyses were conducted using SPSS Statistics Version 23.0. Statistical assumptions for each analysis were considered prior to data analyses, and all assumptions were met. Past studies have found that age and gender are significant predictors of one's psychological well-being (Soysa \& Wilcomb, 2013; Stone et al., 2010). Hence, preliminary analyses were also conducted to examine whether the main study variables vary as a function of participants' gender and age. Independent sample t-tests showed that participants' self-compassion, anxiety, and affective states did not differ as a function of gender. However, Pearson correlation analyses showed that age was positively associated with self-compassion $(r=0.26$, $p<0.001)$ and negatively associated with more report of negative emotions $(r=-0.19, p<0.001)$. Thus, participants' age was entered as a control variable in subsequent analyses.

\section{Results}

Frequency analysis showed that around one-third (36.6\%) of the participants' anxiety scores reached the recommended clinical significance cut-off point (i.e., 36) for SAS (Dunstan $\&$ Scott, 2020), suggesting greater attention to the general publics' mental health is timely in the pandemic context.
Descriptive statistics and partial correlations between the main study variables after controlling participants' age are presented in Table 1. As predicted, participants' selfcompassion was negatively associated with self-reported anxiety and negative emotions, with medium-to-large effect sizes obtained (Cohen, 1988). The moderate-to-strong associations between self-compassion and individuals' negative affect were consistent with the findings reported in Zessin et al. (2015)'s meta-analysis, suggesting that self-compassion-based intervention may be a plausible strategy to alleviate people's negative affect.

\section{Study 2}

With a significantly negative association established between individuals' self-compassion and negative affect, in study 2 , we investigated the preliminary effectiveness of two brief online self-compassion exercises (i.e., meditation and writing) to reduce the participants' negative affect in the context of pandemic lockdown.

\section{Study 2a}

In study $2 \mathrm{a}$, we delivered an instructor-guided self-compassion meditation to a group of young adults at the end of March 2020 (i.e., when the Chinese public's worrying emotion regarding COVID-19's impact on various life aspects continually increases; Li et al., 2020). Drawing the conclusions from past research, we hypothesized that participants' level of anxiety and negative emotions would be reduced after practicing self-compassion meditation.

\section{Method}

\section{Participants}

Participants were recruited online from social media sites (i.e., QQ and WeChat). The participants were 64 young adults $\left(M_{\text {age }}=26.53, S D=6.15\right)$ between the age of 18 and 45 years old from eighteen provinces in China. In particular, 27 participants self-identified as males $\left(M_{\text {age }}=26.37\right.$, $S D=6.36), 37$ as females $\left(M_{\text {age }}=26.65, S D=6.09\right)$. Most participants reported holding at least a bachelor's degree
Table 1 Descriptive statistics and interrelations between main study variables in study 1

\begin{tabular}{lllllll}
\hline & $M$ & $S D$ & Range & 1 & 2 & 3 \\
\hline 1. Self-compassion & 3.32 & .54 & $1.62-4.92$ & 1 & \\
2. Anxiety & 33.62 & 7.31 & $20-60$ & $-.52^{* * *}$ & 1 & \\
3. Negative emotions & 19.41 & 6.66 & $10-50$ & $-.37^{* * *}$ & $.52^{* * *}$ & 1 \\
\hline
\end{tabular}

Note. ${ }^{* * *} p<.001$. Raw means and standard deviations are presented. Participant age was entered as a covariate in the correlational analyses 
(95.4\%) and with no religious affiliation (98.4\%). While 39 participants $(60.9 \%)$ reported being full-time students, 25 participants $(39.1 \%)$ reported being employed/selfemployed. The majority of the participants (96.9\%) believed that the pandemic impacted them, of which $62.5 \%$ reported a mild impact on them, and $34.4 \%$ reported having a moderate to severe impact on them.

\section{Procedure}

After participants signed the electronic consent form, they were directed to an online survey which includes demographic questions and the state anxiety and negative emotion measures. The survey generally takes 5-10 min to complete. The participants were then invited to join a live session on the Tencent Meeting software, where the self-compassion meditation was delivered. After the meditation training was completed, participants were asked to complete the survey questionnaires again. At the end of the session, participants were thanked for their participation and informed that professional psychological services were also available via telephone if they need any relevant resources and psychological support.

Self-compassion Induction The researcher WR, who is also an experienced mindfulness meditation instructor, guided the self-compassion induction process. To induce participants' self-compassion, the instructor incorporated Neff (2003b)'s three main components of self-compassion: self-kindness, common humanity, and mindfulness in the meditation practice. Specifically, participants were guided to understand that negative emotions are common to all people experiencing pandemic lockdown (common humanity). Participants were also instructed to think about three positive words that show love and caring to themselves (selfkindness) and practice mindful breathing (mindfulness). The online group meditation session lasted $20 \mathrm{~min}$. During the meditation, participants can only hear the instructor' voice, with no visual image available.

\section{Measures}

State Anxiety The State-Trait Anxiety Inventory (STAI; Spielberger et al., 1983) was used to examine individuals' anxiety levels. For the current study - to examine how individuals felt at the moment of pandemic lockdown, only the 20-item "state anxiety" subscale (STAI-S) was used. The STAI-S assesses the degree of individuals' feelings at a particular moment in time. Participants were asked to rate how they felt on a 4-point scale, ranging from $1=$ "not at all/ almost never" to $4=$ "very much so/almost always." Item scores were summed together to generate a total score for the STAI-S. The total score ranges from 20 to 80 , with a higher score indicating more severity in anxiety. The Chinese version of STAI-S used in the current study was translated by $\mathrm{Fu}$ (1997). Excellent internal consistencies for the STAI-S subscale were found in both the pre-test $(\alpha=0.94)$ and posttest analysis $(\alpha=0.94)$.

Negative Emotions The PANAS-NA was also used to assess individuals' negative moods or affective states. However, in study $2 \mathrm{a}$, participants were asked to rate how much they were feeling "in the present moment." Internal consistencies were satisfactory in the current sample before $(\alpha=0.70)$ and after the self-compassion induction $(\alpha=0.78)$.

\section{Data Analyses}

Data analyses were conducted using SPSS Statistics Version 23.0, and relevant statistical assumptions were checked. Preliminary analyses showed that participants' negative emotions and anxiety did not differ as a function of gender in the pre- and post-meditation conditions. Age was only significantly associated with individuals' anxiety level before the meditation $(r=-0.38, p=0.002)$. Thus, participants' gender was not considered as a control variable in subsequent analyses.

\section{Results}

The results of the paired t-tests are presented in Table 2. As can be seen, after the self-compassion meditation, there was a significant decrease in participants' emotional intensity of reported negative emotions. In addition, there was also a significant decrease in participant's anxiety levels (even after controlling age using a repeated-measures ANCOVA, $F(1$, $62)=29.37, p<0.001$ ). These findings provided support to the effectiveness of telehealth delivery of self-compassionbased meditation training in mitigating individuals' negative affect during the pandemic. Telehealth services have been suggested to be particularly critical remote technologies that can screen, care, train, and treat individuals' mental health issues without the risk of exposing oneself to deadly infectious diseases (Young \& Schneider, 2020). Nevertheless, the delivery of meditation training still relies on trained professionals, which may only be accessible to a small number

Table 2 Means and SDs of anxiety and negative emotion before and after self-compassion induction in study $2 \mathrm{a}$

\begin{tabular}{lrrrrrr}
\hline & \multicolumn{2}{l}{ Pre-test } & & \multicolumn{2}{l}{ Post-test } & \multirow{2}{*}{$t$} \\
\cline { 2 - 3 } & Mean & SD & & Mean & SD & \\
\hline State anxiety & 45.14 & 11.13 & & 34.92 & 10.37 & $8.87^{\text {**** }}$ \\
Negative emotions & 22.81 & 8.25 & & 13.94 & 6.34 & $10.96^{* * *}$ \\
\hline
\end{tabular}

Note. ${ }^{* * *} p<.001$. Bootstrapping with 5000 resamples were applied 
of individuals. It is important to investigate more reliable methods that are easy to administer and approachable to the general public.

\section{Study 2b}

As discussed earlier, the desire to handle the problem on one's own was one of the most common reasons people do not seek psychological help, even among individuals who recognize their psychological problems (Mojtabai et al., 2011). Under the circumstances of limited mental health resources and the inconvenience accompanied by social distancing implementation, self-directed internet interventions may be a particularly attractive solution to meet the general public's emotional and psychological needs (Beshai et al., 2020). Thus, in Study 2b, we examined the potential utility of an informal self-compassion exercise (i.e., selfguided writing) on reducing individuals' negative affect in early April 2020. We expected that participants in the selfcompassion induction group who completed the self-guided writing task would report significantly higher self-compassion and lower levels of anxiety and negative emotions than participants in the control group.

\section{Method}

\section{Participants}

Seventy-nine participants (31 males, 48 females; $\left.M_{\text {age }}=31.10, S D=10.19\right)$ from 40 cities of China joined this study. In particular, 40 participants $\left(M_{\text {age }}=35.63\right.$, $S D=10.55)$ were allocated to the self-compassion writing induction group and 39 participants $\left(M_{\text {age }}=26.46\right.$, $S D=7.43$ ) were allocated to the writing control group. All except 4 reported having no religious affiliation.

\section{Procedure}

This study adopted a two-group post-test only randomized experimental design. After signing up for this study, participants were randomly allocated to one of two experimental conditions: (1) self-compassion induction condition and (2) control condition. Then, the participants in each condition were invited to join a live session on the Tencent Meeting software. Following informed consent, participants started to complete the survey, which they were first asked to think about a negative event that they had experienced after the pandemic outbreak. Then, participants were asked to either write down the experience (control) or respond to questions about the negative experiences (self-compassion) depending on their allocated conditions. After the writing tasks, participants were directed to the survey containing the outcome measures (SCS, STAI-S, PANAS). At the end of the session, participants were thanked and received a small amount of money for their participation.

Self-compassion Induction To induce participants' selfcompassion, a self-compassion writing task was used. Participants were asked to think about a negative event that they had experienced after the pandemic outbreak that made them criticize or feel bad about themselves (or any difficult experience that made them feel painful or anxious). Following Leary (2007), participants in the self-compassion condition were then asked to respond to three prompts that guided them to think about the event in a self-compassion way. Each prompt was designed to induce the participants' sense of common humanity, self-kindness, and mindfulness. Specifically, participants were first asked to "list ways in which other people also experience similar events" (common humanity). Then, participants were asked to "write a paragraph expressing understanding, kindness and concern to themselves in the same way that they might express concern to a friend who had undergone the experience" (selfkindness). Finally, participants were asked to "describe their feelings about the event in an objective and unemotional fashion" (mindfulness).

Control Condition Participants in the control condition were asked to think about a negative event that they experienced after the pandemic outbreak and describe the event and their feelings at the time.

\section{Measures}

Self-compassion Participants' compassion towards themselves was assessed using the SCS. In this study, Cronbach's $\alpha$ was 0.87 .

State Anxiety Participants' state anxiety was examined using the STAI-S measure that has been used in study $2 \mathrm{a}$. In this study, Cronbach's $\alpha$ was 0.92 .

Negative Emotions Participants' negative emotions were examined using the PANAS-NA that described in study 2a. Cronbach's $\alpha$ was 0.91 in this study.

\section{Data Analyses}

Data analyses were conducted using SPSS Statistics Version 23.0, and statistical assumptions were checked. Preliminary analyses showed that the main study variables did not differ as a function of age or gender in both the self-compassion and the control group. Hence, participants' age and gender were not included as covariates in subsequent analyses. 


\section{Results}

The results of independent sample t-tests are presented in Table 3. As can be seen, participants in the self-compassion writing condition reported significantly higher perception of self-compassion than participants in the control condition. Accordingly, participants in the self-compassion condition showed a lower level of state anxiety and fewer negative emotions than the participants in the control condition (except the difference for negative emotions did not reach statistical significance). The results provided support to past studies suggesting self-compassion writing can effectively reduce individuals' state anxiety (Harwood \& Kocovski, 2017; Wong \& Mak, 2016).

\section{Study 3}

The results of study $2 \mathrm{a}$ and $2 \mathrm{~b}$ showed a potentially promising effect of the self-compassion intervention on the mitigation of the general public's negative affect in a pandemic context. While these findings were encouraging, the main limitation of study 2 was the lack of pre-test measures in study $2 b$, which result in the effect of the self-compassion writing exercise cannot be accurately estimated. Therefore, we refined the experimental methods of study $2 b$ with larger sample size and pre-test measures. We hypothesized that participants in the self-compassion writing group would report significantly higher self-compassion levels, lower levels of state anxiety, and negative emotions than the control group after the intervention.

\section{Method}

\section{Participants}

Before conducting the study, a priori power analysis was performed using the G*Power 3.1.9.2 (Faul et al., 2007) to determine the sample size required to detect an effect of the self-compassion intervention. Based on the medium effect sizes we obtained in study 2, an ANOVA F-test was carried out with an estimated medium effect size $f=0.25$ (Cohen, 1988), $\alpha=0.05$, power of 0.95 , number of groups $=2$, and number of measurements $=2$. The results showed that a total sample of no fewer than 54 participants was required. One hundred and three college students participated in the study. After excluding participants who did not complete the writing task and who failed the compliance check ( 3 in the self-compassion group and 5 in the control group), 95 participants $\left(M_{\text {age }}=18.63, S D=0.75,68\right.$ males, 27 females $)$ were retained for the data analyses. All except 4 reported having no religious affiliation. Specifically, the self-compassion intervention group consisted of 50 participants $\left(M_{\text {age }}=18.52, S D=0.61\right)$, whereas the control group was composed of 45 participants $\left(M_{\text {age }}=18.76, S D=0.86\right)$.

\section{Procedure}

Participants were recruited from Xihua University, Chengdu, at the end of December 2020 (i.e., when multiple regions in Chengdu were rated as medium-risk areas as a result of several newly confirmed cases). Before the study began, participants were randomly allocated to one of two experimental conditions: (1) self-compassion writing induction condition and (2) neutral control condition. The experiments were carried online via the Tencent Meeting software. After the informed consent was obtained, participants completed a brief survey which first asked them to think about a particular situation that they were experiencing as difficult and indicate how difficult the situation was. Participants were also asked to provide basic demographic information and respond to the pre-test measures (i.e., SSCS-L, PANAS-NA, STAI-S) regarding the situation. Then, participants (both the self-compassion writing group and the control group) were asked to follow the survey's instruction to complete a writing task about the same situation. Participants were reassured that their responses would be anonymous and confidential. After completing the writing tasks, participants answered the compliance check question and filled out the post-test measures. Participants who completed the study were thanked and received monetary compensation (¥30) for their participation.

Self-compassion Induction Following the procedure (i.e., Self-Compassionate Mindstate Induction; SCMI) adopted in Neff et al. (2020)'s research, participants were randomly assigned to a self-compassion writing condition and a control condition. First, all participants were asked to think
Table 3 Means and sds of main study variables by group condition in study $2 b$

\begin{tabular}{|c|c|c|c|c|c|c|}
\hline & \multicolumn{2}{|c|}{ Compassion } & \multicolumn{2}{|c|}{ Control } & \multirow[t]{2}{*}{$t$} & \multirow[t]{2}{*}{ Cohen's $d$} \\
\hline & Mean & SD & Mean & SD & & \\
\hline Self-compassion & 3.19 & .45 & 2.97 & .43 & $2.22^{*}$ & .50 \\
\hline State anxiety & 37.85 & 9.69 & 42.67 & 9.94 & $-2.18^{*}$ & .49 \\
\hline Negative emotions & 16.25 & 5.47 & 18.13 & 6.70 & -1.37 & .31 \\
\hline
\end{tabular}

Note. ${ }^{*} p<.05$. Bootstrapping with 5000 resamples were applied 
about a situation that they were experiencing at the time of data collection that was painful or difficult. After deciding the single situation that they would focus on throughout the study, participants indicated on a 5-point Likert scale (from $1=$ "a little difficult" to $5=$ "extremely difficult") about how difficult the situation they felt. Most participants chose a fairly difficult $(M=3.40, S D=1.06)$ situation to think about.

Self-compassion Writing Condition Participants assigned to the self-compassion intervention group completed a writing task that contained a series of writing prompts that aimed to induce the three components of self-compassion: mindfulness, common humanity, and self-kindness. The SCMI instruction was first translated by two bilingual researchers, FG and YW, and then inspected by the researchers involved in this study before the final version was established (see Appendix $C$ in the supplementary materials for the translated instruction). While preserving the literal nature of the translation, we noticed that the example provided for the "writing prompt" does not suit the Chinese culture (i.e., "I feel frustrated about the fact that my mom doesn't understand why I don't want to come home for Thanksgiving..."). Therefore, we have replaced the "Thanksgiving" with a Chinese national holiday, "The Dragon Boat Festival."

Neutral Control Condition The control condition was designed to be parallel to the SCMI. Following Neff's procedures, the participants who were assigned to the control condition were asked to first write about the painful situation in a descriptive manner (parallel to mindfulness). The participants were then asked to specify the individuals that were involved (parallel to common humanity) and describe any words spoken in the situation (parallel to self-kindness).

Compliance Check Finally, all participants were asked to indicate what they had just been asked to do during the research session. Specifically, participants were required to select (a) "write about your feelings in an accepting and validating way, consider how going through difficult situations is part of being human, write to yourself like a supportive friend" or (b) "write the details of the situation, who is involved and what was said with as much detail as possible." Those in the self-compassion writing condition passed the compliance check if they responded "a" and the controls if they responded "b."

\section{Measures}

State Self-compassion Scale-Long Form The 18-item state self-compassion scale-long form (SSCS-L; Neff et al., 2020) was used to assess the participants' self-compassion level at the time of data collection. An example item includes "I'm keeping my emotions in balanced perspective." Participants rated each item on a 5-point Likert scale, ranging from $1=$ "not at all true for me" to $5=$ "very true for me." Before generating an overall compassion score, items representing self-judgment, isolation, and over-identification were reverse-coded. Then, the mean score of the 18 items was calculated, where higher scores represented greater degrees of state self-compassion. The SSCS-L has been shown to have robust psychometrical properties (Neff et al., 2020). The Chinese version of the SSCS-L was translated and backtranslated by two bilingual researchers involved in this study, FG and YW (see Appendix B in the supplementary materials for the translated scale). In this study, the scale showed good internal reliabilities both pre-test $(\alpha=0.78)$ and posttest $(\alpha=0.84)$.

State Anxiety The STAI-S was used to examine participants' state anxiety. In this study, Cronbach's $\alpha$ was 0.92 at pre-test and was 0.93 at post-test.

Negative Emotions Participants' negative emotions were examined using the PANAS-NA. The PANAS-NA was found to be reliable at pre-test $(\alpha=0.84)$ and at post-test $(\alpha=0.89)$.

\section{Results}

Table 4 presents the means and standard deviations of the main study measures at pre-test and post-test. There were no statistically significant differences between the selfcompassion writing group and control group on all study variables at pre-test (all $p s>0.05$ ). The results of the paired $\mathrm{t}$-test of pre/post-test outcomes showed that there were substantial increases in participants' level of self-compassion
Table 4 Pre-test and post-test mean scores by condition and self-compassionate writing intervention effects analyzed with 2 (Condition) $\times 2$ (Time) repeated measures analyses of variance in study 3

\begin{tabular}{|c|c|c|c|c|c|c|}
\hline \multirow[t]{2}{*}{ Outcome } & \multicolumn{2}{|c|}{$\begin{array}{l}\text { Self-compassionate writing condi- } \\
\text { tion }\end{array}$} & \multicolumn{2}{|c|}{ Neutral control condition } & \multirow[t]{2}{*}{$F$} & \multirow[t]{2}{*}{$\eta_{p}^{2}$} \\
\hline & Pre-test $M(S D)$ & Post-test $M(S D)$ & Pre-test $M(S D)$ & Post-test $M(S D)$ & & \\
\hline Self-compassion & $3.45(.63)$ & $3.76(.67)$ & $3.48(.42)$ & $3.51(.45)$ & $7.04^{* *}$ & .07 \\
\hline State anxiety & $53.16(1.70)$ & $42.44(1.58)$ & $52.33(1.79)$ & $51.24(1.67)$ & $13.18^{* * *}$ & .12 \\
\hline Negative emotions & $23.58(1.13)$ & $17.88(1.03)$ & $22.42(1.19)$ & $20.56(1.09)$ & $7.46^{* * *}$ & .07 \\
\hline
\end{tabular}


$(t=-5.14, p<0.001)$, and decreases in negative emotions $(t=6.87, p<0.001)$ and anxiety $(t=5.58, p<0.001)$ in the self-compassion writing group. The neutral control group also demonstrated a slight increase in participants' level of self-compassion $(t=-0.35)$ and decreases in participants' negative emotions $(t=1.61)$ and anxiety $(t=0.60)$. However, none of the pre/post-test changes in the control condition reached statistical significance (all $p s>0.05$ ).

To determine whether there was a greater degree of mitigation on participants' negative affect in the self-compassion writing group, we performed $2 \times 2$ repeated-measures ANOVA with Condition (self-compassion and control) as a between-subjects factor and Time (pre-test and post-test) as a within-subjects factor. As can be seen in Table 4, compared to the participants in the control condition, participants in the self-compassion writing condition demonstrated significantly greater gains in self-compassion and more reduction in their level of anxiety and negative emotions.

Again, these results align with prior research findings suggesting self-compassion writing could reduce individuals' negative emotions and anxiety (Harwood \& Kocovski, 2017; Odou \& Brinker, 2014; Wong \& Mak, 2016). With the further improvement of the intervention design in study 3 , these results provided more robust evidence that self-compassion-based practices can be effective tools to mitigate the public's negative affect in a pandemic context through online delivery.

\section{Discussion}

In the present investigation, we aimed to establish relations between self-compassion and negative affect among Chinese citizens and investigate the potential utility of online selfcompassion exercises on reducing the negative affect in the context of the pandemic lockdown. As expected in study 1, we found a significant negative association between individuals' self-compassion perception and negative affect (anxiety and negative emotions). Furthermore, the two pilot studies (study $2 \mathrm{a}$ and $2 \mathrm{~b}$ ) revealed that online self-compassion exercises (both instructor-guided meditation and self-guided writing) might have a promising effect on alleviating anxiety and reducing negative emotions among the general public. Last, study 3 further provided support for the effectiveness of self-compassion writing exercise to mitigate individuals' negative affect.

In keeping with our first hypothesis, the finding (study 1) that individuals with high self-compassion perception tended to report less negative affect suggested that self-compassion as a healthy self-attitude may help promote people's emotional well-being. The significant negative associations with medium effect sizes were consistent with prior research studies, which found moderate-to-strong relations between self-compassion and various forms of well-being in Western industrialized societies (Neff \& Dahm, 2017; Nelson et al., 2016; Zessin et al., 2015). For example, prior research has found that higher self-compassion was associated with less depression, anxiety, rumination, thought suppression, and greater life satisfaction and social relatedness (Neff, 2003b). The finding suggests that both in Western and Eastern culture, self-compassion is a robust predictor of anxious symptomatology and emotional well-being.

In the current pandemic, many regions across the world have implemented strict social distancing measures, such as city lockdown, school and public facilities closure, and stay-at-home instruction for the general public. Online delivery of self-compassion interventions may be promising in reaching as many impacted citizens as possible. Our findings (study $2 \mathrm{~b}$ and study 3 ) showed that online self-compassion exercises could successfully induce individuals' self-compassion. Moreover, similar to past research that has investigated the effectiveness of telehealth delivery of selfcompassion interventions, our results revealed that inducing self-compassion (even in a brief session) can reduce anxiety (study 2 and study 3 ) and negative emotions (study 2a and study 3) (Finlay-Jones et al., 2017; Halamová et al., 2018; Krieger et al., 2019; Rao \& Kemper, 2016). Self-compassion involves unconditional self-worth, which entails compassion for ourselves even when we face distress and difficulties (Neff, 2003b). Self-compassion coping/intervention, therefore, may represent a healthier form of self-acceptance and plays a vital role in how people cope with emotional problems (Friis et al., 2016; Karanika \& Hogg, 2016; Liu et al., 2020). These results suggested that self-compassion may have advantages both as a predictor and an indicator for negative public affect in the current outbreak of the COVID19 pandemic.

As mentioned earlier, despite that the concept of selfcompassion was drawn from Eastern Buddhism philosophy, there is a lack of empirical study examining the association between self-compassion and individuals' emotional well-being in Eastern countries (Neff \& Dahm, 2017; Neff, 2003b; Nelson et al., 2016). Hence, our findings are important as they provided empirical evidence to the notion that self-compassion is vital to individuals' psychological wellbeing in an Eastern Asian context (i.e., China). Furthermore, as the pandemic continues to spread, it is clear that a large number of people have experienced or are experiencing varying degrees of emotional distress (Li et al., 2020). Our findings showed that self-compassion exercises were effective to alleviate individuals' negative affect. Of importance, the finding that participants' anxiety levels were significantly lower for participants who joined the self-compassion writing exercise than the controls showed that self-directed compassionate training could be a promising method to meet the general public's emotional and psychological needs. 
The online self-guided form of self-compassion exercise may overcome the common barriers (e.g., financial difficulties, desires to handle the issue by oneself) which discourage people from seeking mental health care and solve the mental health resources and transportation limitation issues caused by the pandemic lockdown (Andrade et al., 2014; Beshai et al., 2020).

\section{Limitations and Future Research}

Some limitations of this study are worth noting. First, given that our study used the self-report survey data based on same-respondent replies, common method variance resulting from participants' social desirable responses and implicit theories may be a concern (Podsakoff et al., 2003). Second, our findings were mainly based on the results of single brief intervention sessions. It is unclear about the long-term effectiveness of these self-compassion exercises on individuals' emotional well-being. Future studies could examine the self-compassion interventions using a longitudinal design to estimate and confirm their effects on people's negative affect and explore their potential benefit on other forms of psychological well-being. Third, the samples included in study 2 were small, and there was a lack of sociodemographic information of the participants. It is less clear about the generalizability of current findings to a wider population. Future research could assess the effectiveness of these selfcompassion exercises in a broader and more representative sample. Moreover, given self-compassion is an Eastern philosophy, it is possible that participants from Eastern cultures may be more receptive to self-compassion-based training. In addition, most participants included in our studies were non-religious. Thus, future studies could examine the applicability of online self-compassion exercises in the general public from countries with different cultural backgrounds and religions.

Fourth, we did not include a control group in study $2 \mathrm{a}$. It is possible that this methodological limitation may affect the results that were obtained in study $2 \mathrm{a}$. Future studies on this topic could include a control group to test whether these findings could be replicated. Finally, our study only assessed the effect of self-compassion practice as a whole. It is unclear which of the three self-compassion components contribute most to the mitigation of individuals' negative affect. Future studies could compare the effect of three separate practices (each inducing only one component of selfcompassion) with a control group to determine whether the effect of self-compassion practice is mainly driven by one component or equally by all of the components.

Supplementary Information The online version contains supplementary material available at https://doi.org/10.1007/s12671-021-01674-w.
Author Contribution FG and YW: contributed equally to this paper and shared co-first authorship. FG, YW, KPP and JBH: conceptualized the study, performed data analyses, and wrote and revised the paper. PZ, WR: collaborated with the design and assisted with data collection and analyses. BJ, ZX, STW: collaborated with the design, executed the study, and collaborated to write and edit the final manuscript. All authors approved the final version of the manuscript for submission.

Funding This study was supported by the Tsinghua University Spring Breeze Fund (2020Z99CFG013).

\section{Declarations}

Ethical Standards This study was performed in line with the principles of the Declaration of Helsinki. Approval was granted by the Research Ethics Committee of the Department of Psychology at Tsinghua University (Date: 28/02/2020/No.: 2020/27).

Informed Consent Statement All participants gave their informed consent prior to their inclusion in the study.

Conflict of Interest The authors declare no competing interests.

\section{References}

Andrade, L. H., Alonso, J., Mneimneh, Z., Wells, J. E., Al-Hamzawi, A., Borges, G., Bromet, E., Bruffaerts, R., de Girolamo, G., de Graaf, R., Florescu, S., Gureje, O., Hinkov, H. R., Hu, C., Huang, Y., Hwang, I., Jin, R., Karam, E. G., Kovess-Masfety, V., ... Kessler, R. C. (2014). Barriers to mental health treatment: Results from the WHO world mental health surveys. Psychological Medicine, 44(6), 1303-1317. https://doi.org/10.1017/s0033 291713001943

Barnard, L. K., \& Curry, J. F. (2011). Self-compassion: Conceptualizations, correlates, \& interventions. Review of General Psychology, 15(4), 289-303. https://doi.org/10.1037/a0025754

Beshai, S., Bueno, C., Yu, M., Feeney, J. R., \& Pitariu, A. (2020). Examining the effectiveness of an online program to cultivate mindfulness and self-compassion skills (Mind-OP): Randomized controlled trial on Amazon's Mechanical Turk. Behaviour Research and Therapy, 134, 103724. https://doi.org/10.1016/j. brat.2020.103724

Bluth, K., \& Eisenlohr-Moul, T. A. (2017). Response to a mindful self-compassion intervention in teens: A within-person association of mindfulness, self-compassion, and emotional well-being outcomes. Journal of Adolescence, 57, 108-118. https://doi.org/ 10.1016/j.adolescence.2017.04.001

Cohen, J. (1988). Statistical power analysis for the behavioral sciences. Routledge Academic.

Coyne, L. W., Gould, E. R., Grimaldi, M., Wilson, K. G., Baffuto, G., \& Biglan, A. (2020). First things first: Parent psychological flexibility and self-compassion during COVID-19. Behavior Analysis in Practice, 1-7https://doi.org/10.1007/s40617-020-00435-w

Dua, J. K. (1993). The role of negative affect and positive affect in stress, depression, self-esteem, assertiveness, type A behaviors, psychological health, and physical health. Genetic, Social, and General Psychology Monographs, 119(4), 515-552.

Dundas, I., Binder, P. E., Hansen, T. G. B., \& Stige, S. H. (2017). Does a short self-compassion intervention for students increase healthy self-regulation? A randomized control trial. Scandinavian Journal of Psychology, 58(5), 443-450. https://doi.org/10.1111/ sjop. 12385 
Dunstan, D. A., \& Scott, N. (2020). Norms for Zung's self-rating anxiety scale. BMC Psychiatry, 20https://doi.org/10.1186/ s12888-019-2427-6

Faul, F., Erdfelder, E., Lang, A.-G., \& Buchner, A. (2007). G*Power 3: A flexible statistical power analysis program for the social, behavioral, and biomedical sciences. Behavior Research Methods, 39(2), 175-191. https://doi.org/10.3758/BF03193146

Finlay-Jones, A., Kane, R., \& Rees, C. (2017). Self-compassion online: A pilot study of an internet-based self-compassion cultivation program for psychology trainees. Journal of Clinical Psychology, 73(7), 797-816. https://doi.org/10.1002/jclp.22375

Friis, A. M., Johnson, M. H., Cutfield, R. G., \& Consedine, N. S. (2016). Kindness matters: A randomized controlled trial of a mindful self-compassion intervention improves depression, distress, and HbA1c among patients with diabetes. Diabetes Care, 39(11), 1963-1971. https://doi.org/10.2337/dc16-0416

$\mathrm{Fu}$, J. (1997). Confirmatory factor analysis of the state-trait anxiety inventory. Chinese Mental Health Journal, 11(4), 216-217.

Grinde, B. (2015). Why negative feelings are important when assessing well-being. Journal of Happiness Studies, 17, 1741-1752. https:// doi.org/10.1007/s10902-015-9667-z

Gupta, V. K., Singh, A., Amish, \& Chauhan, R. (2020). Can selfcompassion be a resilient factor for psychological distress? Relationship of self-compassion with psychological distress during lockdown due to novel coronavirus disease (COVID-19) outbreak in India. Journal of Critical Reviews, 7(13), 2551-2561. https:// doi.org/10.31838/jcr.07.13.391

Halamová, J., Kanovský, M., Varšová, K., \& Kupeli, N. (2018). Randomised controlled trial of the new short-term online emotion focused training for self-compassion and self-protection in a nonclinical sample. Current Psychology, 40, 333-343. https://doi.org/ 10.1007/s12144-018-9933-4

Harwood, E. M., \& Kocovski, N. L. (2017). Self-compassion induction reduces anticipatory anxiety among socially anxious students. Mindfulness, 8(6), 1544-1551. https://doi.org/10.1007/ s12671-017-0721-2

Huang, Y., \& Zhao, N. (2020). Generalized anxiety disorder, depressive symptoms and sleep quality during COVID-19 outbreak in China: A web-based cross-sectional survey. Psychiatry Research, 288, 112954-112954. https://doi.org/10.1016/j.psychres.2020.112954

Jiménez, Ó., Sánchez-Sánchez, L. C., \& García-Montes, J. M. (2020). Psychological impact of covid-19 confinement and its relationship with meditation. International Journal of Environmental Research and Public Health, 17(18), 6642. https://doi.org/10. 3390/ijerph17186642

Karanika, K., \& Hogg, M. K. (2016). Being kind to ourselves: Selfcompassion, coping, and consumption. Journal of Business Research, 69(2), 760-769. https://doi.org/10.1016/j.jbusres.2015. 07.042

Kavakli, M., M, A. K., Uğuz, F., \& Türkmen, O. (2020). The mediating role of self-compassion in the relationship between perceived COVID-19 threat and death anxiety. Turkish Journal of Clinical Psychiatry, 23, 15-23. https://doi.org/10.5505/kpd.2020.59862

Krieger, T., Reber, F., von Glutz, B., Urech, A., Moser, C. T., Schulz, A., \& Berger, T. (2019). An internet-based compassion-focused intervention for increased self-criticism: A randomized controlled trial. Behavior Therapy, 50(2), 430-445. https://doi.org/10.1016/j. beth.2018.08.003

Langarizadeh, M., Tabatabaei, M. S., Tavakol, K., Naghipour, M., Rostami, A., \& Moghbeli, F. (2017). Telemental health care, an effective alternative to conventional mental care: A systematic review. Acta Informatica Medica, 25(4), 240-246. https://doi.org/ 10.5455/aim.2017.25.240-246

Lau, B., Chan, C., \& Ng, S.-M. (2020). Self-compassion buffers the adverse mental health impacts of COVID-19-related threats: Results from a cross-sectional survey at the first peak of Hong
Kong's outbreak. Frontiers in Psychology, 11, 1203. https://doi. org/10.31234/osf.io/5vdpz

Leary, M. R. (2007). Motivational and emotional aspects of the self. Annual Review of Psychology, 58, 317-344. https://doi.org/10. 1146/annurev.psych.58.110405.085658

Li, X., Zhou, M., Wu, J., Yuan, A., Wu, F., \& Li, J. (2020). Analyzing COVID-19 on online social media: Trends, sentiments and emotions. ArXiv. abs/2005.14464

Liu, G., Zhang, N., Teoh, J. Y., Egan, C., Zeffiro, T. A., Davidson, R. J., \& Quevedo, K. (2020). Self-compassion and dorsolateral prefrontal cortex activity during sad self-face recognition in depressed adolescents. Psychological Medicine, 1-10https://doi.org/10.1017/ s0033291720002482

Mak, I. W. C., Chu, C. M., Pan, P. C., Yiu, M. G. C., \& Chan, V. L. (2009). Long-term psychiatric morbidities among SARS survivors. General Hospital Psychiatry, 31(4), 318-326. https://doi. org/10.1016/j.genhosppsych.2009.03.001

Mann, F. D., Krueger, R. F., \& Vohs, K. D. (2020). Personal economic anxiety in response to COVID-19. Personality and Individual Differences, 167, 110233-110233. https://doi.org/10.1016/j.paid. 2020.110233

Mojtabai, R., Olfson, M., Sampson, N. A., Jin, R., Druss, B., Wang, P. S., Wells, K. B., Pincus, H. A., \& Kessler, R. C. (2011). Barriers to mental health treatment: Results from the national comorbidity survey replication. Psychological Medicine, 41(8), 1751-1761. https://doi.org/10.1017/s0033291710002291

Neff, K. D. (2003a). The development and validation of a scale to measure self-compassion. Self and Identity, 2(3), 223-250. https:// doi.org/10.1080/15298860309027

Neff, K. D. (2003b). Self-compassion: An alternative conceptualization of a healthy attitude toward oneself. Self and Identity, 2(2), 85-101. https://doi.org/10.1080/15298860309032

Neff, K. D. (2016). The self-compassion scale is a valid and theoretically coherent measure of self-compassion. Mindfulness, 7(1), 264-274. https://doi.org/10.1007/s12671-015-0479-3

Neff, K. D., \& Dahm, K. A. (2017). Self-compassion: What it is, what it does, and how it relates to mindfulness. Routledge/Taylor \& Francis Group.

Neff, K. D., Tóth-Király, I., Knox, M. C., Kuchar, A., \& Davidson, O. (2020). The development and validation of the state self-compassion scale (long- and short form). Mindfulness, 12, 121-140. https://doi.org/10.1007/s12671-020-01505-4

Nelson, S. K., Layous, K., Cole, S. W., \& Lyubomirsky, S. (2016). Do unto others or treat yourself? The effects of prosocial and selffocused behavior on psychological flourishing. Emotion, 16(6), 850-861. https://doi.org/10.1037/emo0000178

Odou, N., \& Brinker, J. (2014). Exploring the relationship between rumination, self-compassion, and mood. Self and Identity, 13(4), 449-459. https://doi.org/10.1080/15298868.2013.840332

Ofri, D. (2009). The emotional epidemiology of H1N1 influenza vaccination. The New England Journal of Medicine, 361(27), 2594. https://doi.org/10.1056/NEJMp0911047

Petzold, M. B., Bendau, A., Plag, J., Pyrkosch, L., Mascarell Maricic, L., Betzler, F., Rogoll, J., Große, J., \& Ströhle, A. (2020). Risk, resilience, psychological distress, and anxiety at the beginning of the COVID-19 pandemic in Germany. Brain and Behavior, 10(9), e01745. https://doi.org/10.1002/brb3.1745

Podsakoff, P. M., MacKenzie, S. B., Lee, J. Y., \& Podsakoff, N. P. (2003). Common method biases in behavioral research: A critical review of the literature and recommended remedies. Journal of Applied Psychology, 88(5), 879-903. https://doi.org/10.1037/ 0021-9010.88.5.879

Racine, N., Hartwick, C., Collin-Vézina, D., \& Madigan, S. (2020). Telemental health for child trauma treatment during and postCOVID-19: Limitations and considerations. Child Abuse \& Neglect, 104698https://doi.org/10.1016/j.chiabu.2020.104698 
Raes, F. (2011). The effect of self-compassion on the development of depression symptoms in a non-clinical sample. Mindfulness, 2, 33-36. https://doi.org/10.1007/s12671-011-0040-y

Rajkumar, R. P. (2020). COVID-19 and mental health: A review of the existing literature. Asian Journal of Psychiatry, 52, 102066102066. https://doi.org/10.1016/j.ajp.2020.102066

Rao, N., \& Kemper, K. J. (2016). Online training in specific meditation practices improves gratitude, well-being, self-compassion, and confidence in providing compassionate care among health professionals. Journal of Evidence-Based Complementary \& Alternative Medicine, 22(2), 237-241. https://doi.org/10.1177/ 2156587216642102

Smeets, E., Neff, K., Alberts, H., \& Peters, M. (2014). Meeting suffering with kindness: Effects of a brief self-compassion intervention for female college students. Journal of Clinical Psychology, 70(9), 794-807. https://doi.org/10.1002/jclp.22076

Soysa, C., \& Wilcomb, C. (2013). Mindfulness, self-compassion, selfefficacy, and gender as predictors of depression, anxiety, stress, and well-being. Mindfulness, 6, 217-226. https://doi.org/10.1007/ s12671-013-0247-1

Spielberger, C., Gorsuch, R., Lushene, R., Vagg, P. R., \& Jacobs, G. (1983). Manual for the state-trait anxiety inventory (form Y1 Y2) (Vol. IV).

Stone, A. A., Schwartz, J. E., Broderick, J. E., \& Deaton, A. (2010). A snapshot of the age distribution of psychological well-being in the United States. Proceedings of the National Academy of Sciences, 107(22), 9985. https://doi.org/10.1073/pnas.1003744107

Wang, C., Pan, R., Wan, X., Tan, Y., Xu, L., McIntyre, R. S., Choo, F. N., Tran, B., Ho, R., Sharma, V. K., \& Ho, C. (2020). A longitudinal study on the mental health of general population during the COVID-19 epidemic in China. Brain, Behavior, and Immunity, 87, 40-48. https://doi.org/10.1016/j.bbi.2020.04.028

Wang, P. S., Aguilar-Gaxiola, S., Alonso, J., Angermeyer, M. C., Borges, G., Bromet, E. J., Bruffaerts, R., de Girolamo, G., de Graaf, R., Gureje, O., Haro, J. M., Karam, E. G., Kessler, R. C., Kovess, V., Lane, M. C., Lee, S., Levinson, D., Ono, Y., Petukhova, M., ... Wells, J. E. (2007). Use of mental health services for anxiety, mood, and substance disorders in 17 countries in the WHO world mental health surveys. Lancet, 370(9590), 841-850. https://doi.org/10.1016/S0140-6736(07)61414-7
Watson, D., Clark, L. A., \& Tellegen, A. (1988). Development and validation of brief measures of positive and negative affect: The PANAS scales. Journal of Personality and Social Psychology, 54(6), 1063-1070. https://doi.org/10.1037//0022-3514.54.6.1063

Whaibeh, E., Mahmoud, H., \& Naal, H. (2020). Telemental health in the context of a pandemic: the covid-19 experience. Current Treatment Options in Psychiatry, 1-5https://doi.org/10.1007/ s40501-020-00210-2

Wilson, A. C., Mackintosh, K., Power, K., \& Chan, S. W. Y. (2019). Effectiveness of self-compassion related therapies: A systematic review and meta-analysis. Mindfulness, 10(6), 979-995. https:// doi.org/10.1007/s12671-018-1037-6

Wong, C. C. Y., \& Mak, W. W. S. (2016). Writing can heal: Effects of self-compassion writing among Hong Kong Chinese college students. Asian American Journal of Psychology, 7(1), 74-82. https://doi.org/10.1037/aap0000041

Wu, W. (1990). Self-rating anxiety scale (SAS). Shanghai Archives of Psychiatry, 2(44).

Yang, Y., Zhang, M., \& Kou, Y. (2016). Self-compassion and life satisfaction: The mediating role of hope. Personality and Individual Differences, 98, 91-95. https://doi.org/10.1016/j.paid.2016.03.086

Young, S. D., \& Schneider, J. (2020). Clinical care, research, and telehealth services in the era of social distancing to mitigate COVID19. AIDS and Behavior, 24(7), 2000-2002. https://doi.org/10. 1007/s10461-020-02924-z

Zessin, U., Dickhäuser, O., \& Garbade, S. (2015). The relationship between self-compassion and well-being: A meta-analysis. Applied Psychology: Health and Well-Being, 7(3), 340-364. https://doi.org/10.1111/aphw.12051

Zung, W. W. (1971). A rating instrument for anxiety disorders Psychosomatics. Journal of Consultation and Liaison Psychiatry, 12(6), 371-379. https://doi.org/10.1016/S0033-3182(71)71479-0

Publisher's Note Springer Nature remains neutral with regard to jurisdictional claims in published maps and institutional affiliations. 